\title{
THE STABILITY OF A STEEL WELDED GIRDER WITH BENDING AND SHEAR FORCES INCLUDED
}

\author{
Ryszard SYGULSKI", Michał GUMINIAK**, Łukasz POLUS** \\ *Polytechnic Faculty, The President Stanisław Wojciechowski Higher Vocational State School in Kalisz, Poznańska 201-205, Kalisz, Poland \\ ${ }^{*}$ Institute of Structural Engineering, Poznan University of Technology, Piotrowo 5, 60-965 Poznan, Poland \\ ryszard.sygulski@gmail.com, michal.guminiak@put.poznan.pl, lukasz.polus@put.poznan.pl
}

received 14 October 2015, revised 22 February 2017, accepted 6 March 2017

\begin{abstract}
The stability of the element of a steel welded girder subjected to bending and shear forces is considered. The considered element is a rectangular plate supported on boundary. The type of a plate boundary conditions depend on the types (thickness) of the stiffeners. Considered plate is loaded by in-plane forces causing bending and shear effects. The Finite Element Method was applied to carry out the analysis. Additionally the Boundary Element Method in terms of boundary-domain integral equation was applied to evaluate the critical shear loading.
\end{abstract}

Key words: Buckling Analysis, the Finite Element Method, the Boundary Element Method

\section{INTRODUCTION AND PROBLEM FORMULATION}

The correct determination of the critical load is essential in the design process. This issue was investigated and solved in an analytical way by Timoshenko et al. (1962). The Finite Element Method (FEM) is applied to a numerical investigation of the stability of the steel welded girder with the bending and the shear forces included. Suitable numerical algorithms of FEM were presented e.g. by Rakowski and Kacprzyk (2005). Others e.g. Shi (1990), Chinnaboon et al. (2007)have used the Boundary Element Method (BEM) to solve the buckling problem of thin plates of any shape including plates with holes. Garstecki and Rzeszut (2009) solved the stability problem of thin walled cold formed sigma profiles with geometrical imperfection. Marcinowski (2007) analysed stability of relatively deep segments of spherical shells loaded by external pressure. The non-linear stability of elastic thin walled structures considering unilateral constraints was investigated by Rzeszut and Garstecki (2011). The direct non-singular formulation of the boundary element method using the fundamental solutions given by Ganowicz and its application to a static analysis of plates with intermediate thickness was presented by Litewka and Sygulski (2010). The analysis of the influence of the fire load on the class of cross-section of steel structural elements was presented by Rzeszut and Polus (2013). The stability problem of thin walled girders considering initial imperfections was investigated by Rzeszut and Garstecki (2013) and Chybinski et al. (2013). Biegus and Kowal (2013) presented that the insufficient buckling strength may lead to disaster. Gosowski (1999) analysed spatial buckling of thin-walled steel-construction beam-columns with discrete bracings. Load capacity and the stability of steel thinwalled beams with local stiffening elements were analysed by Chybiński (2015).

The stability of steel welded girders subjected to in-plane complex loading is presented in the paper. The part of the main structure is indicated in the Fig. 1. The element being a part of the steel welded girder is considered as the square plate, simplysupported with a clamped edge (Fig. 2a) and simply-supported (Fig 2b).

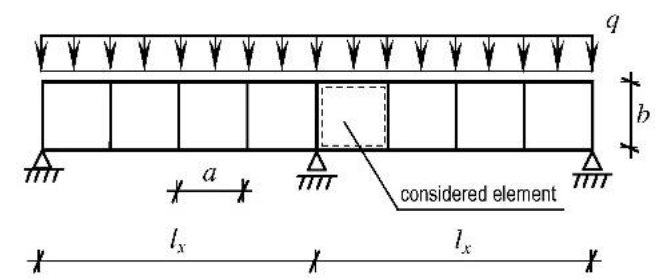

Fig. 1. Steel girder with vertical stiffeners
Girder cross-section

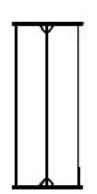

a)

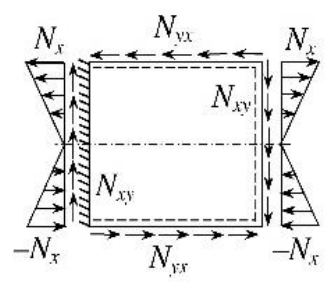

b)

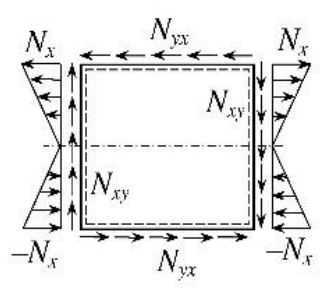

Fig. 2. Considered element

It usually works as a part of the structure under bending and shear load. It is assumed that the material is linear-elastic. The considered plates are subjected to $N_{x}$ and $N_{x y}$ in-plane forces with linear and constant distributions respectively (Fig. 2a and 2b).

\section{AN APPLICATION OF THE FEM}

The differential equation qoverning of plate initial stability has the form: 
$D \cdot \nabla^{4} w=-\bar{p}$,

where $\bar{p}$ is the substitute load

$\bar{p}=N_{x} \cdot \frac{\partial^{2} w}{\partial x^{2}}+2 N_{x y} \cdot \frac{\partial^{2} w}{\partial x \partial y}$.

The Finite Element Method is applied to solve the initial stability problem described by equation (1). In the current analysis four types of finite elements are applied: (i) the rectangular four node shell elements with three degrees of freedom (DOF) per node (deflection and angle of rotations in two perpendicular directions); (ii) the triangular three node shell elements with three DOF per node; (iii) the rectangular eight node shell elements with three DOF per node (deflection and angle of rotations in two perpendicular directions); (iv) and the triangular six node shell elements with three DOF per node. The reduced integration of all shape functions will be applied too. Applying the FEM in pure form leads to the generalized eigenvalue problem (Rakowski and Kacprzyk, 2005)

$\left(K-\lambda \cdot K_{G}\right) \cdot \tilde{q}=0$,

where: $\lambda=N_{c r}$ is critical multiplier, $K$ is the stiffness matrix, $K_{G}$ is the geometric stiffness matrix and $\tilde{q}$ is the vector of nodal displacement with non-zero elements.

The finite element analysis was carried out using the Abaqus system (Abaqus, 2005).

\section{AN APPLICATION OF THE BEM}

The Boundary Element Method in terms of the boundary domain integral equations is introduced. The initial stability problem of the plate subjected only to $N_{x y}$ in-plane forces is solved as the simple benchmark test in reference to the FEM. The solution of differential equation (1) can be expressed as the integral representation of two boundary-domain integral equations formulated according to the simplified approach (Guminiak and Sygulski, 2003; Guminiak, 2014):

$$
\begin{aligned}
& c(\mathrm{x}) \cdot w(\mathrm{x})+\int_{\Gamma}\left[T_{n}^{*}(\mathrm{y}, \mathrm{x}) \cdot w(\mathrm{y})+\right. \\
& \left.-M_{n s}^{*}(\mathrm{y}, \mathrm{x}) \cdot \frac{d w(\mathrm{y})}{d s}-M_{n}^{*}(\mathrm{y}, \mathrm{x}) \cdot \varphi_{n}(\mathrm{y})\right] \cdot d \Gamma(\mathrm{y})= \\
& =\int_{\Gamma}\left[\tilde{T}_{n}(\mathrm{y}) \cdot w^{*}(\mathrm{y}, \mathrm{x})-M_{n}(\mathrm{y}) \cdot \varphi_{n}^{*}(\mathrm{y}, \mathrm{x})\right] \cdot d \Gamma(\mathrm{y})+ \\
& +\int_{\Omega} 2 N_{x y} \cdot \frac{\partial^{2} w}{\partial x \partial y} \cdot w^{*}(\mathrm{y}, \mathrm{x}) \cdot d \Omega(\mathrm{y}) \\
& c(\mathrm{x}) \cdot \varphi_{n}(\mathrm{x})+\int_{\Gamma}\left[\bar{T}_{n}^{*}(\mathrm{y}, \mathrm{x}) \cdot w(\mathrm{y})+\right. \\
& \left.-\bar{M}_{n s}^{*}(\mathrm{y}, \mathrm{x}) \cdot \frac{d w(\mathrm{y})}{d s}-\bar{M}_{n}^{*}(\mathrm{y}, \mathrm{x}) \cdot \varphi_{n}(\mathrm{y})\right] \cdot d \Gamma(\mathrm{y})= \\
& =\int_{\Gamma}\left[\tilde{T}_{n}(\mathrm{y}) \cdot \bar{w}^{*}(\mathrm{y}, \mathrm{x})-M_{n}(\mathrm{y}) \cdot \bar{\varphi}_{n}^{*}(\mathrm{y}, \mathrm{x})\right] \cdot d \Gamma(\mathrm{y})+ \\
& +\int_{\Omega} 2 N_{x y} \cdot \frac{\partial^{2} w}{\partial x \partial y} \cdot \bar{w}^{*}(\mathrm{y}, \mathrm{x}) \cdot d \Omega(\mathrm{y})
\end{aligned}
$$

where the fundamental solution of biharmonic equation:

$\nabla^{4} w^{*}(\mathrm{y}, \mathrm{x})=\frac{1}{D} \cdot \delta(\mathrm{y}, \mathrm{x})$

is given as:

$w^{*}(\mathrm{y}, \mathrm{x})=\frac{1}{8 \pi D} \cdot r^{2} \cdot \ln (r)$

for a thin isotropic plate, $r=|y-x|, \delta$ is the Dirac delta, $D=E h^{3} /\left(12\left(1-v^{2}\right)\right)$ is the plate stiffness, $\mathrm{x}$ is the source point and $\mathrm{y}$ is a field point. The coefficient $c(x)$ is taken as: $c(x)=1$, when $\mathrm{x}$ is located inside the plate domain, $c(x)=$ 0.5 , when $\mathrm{x}$ is located on the smooth boundary and $c(x)=0$, when $\mathrm{x}$ is located outside the plate domain. The second boundary-domain integral equation (3) can be obtained by replacing the unit concentrated force $P^{*}=1$ with the unit concentrated moment $M_{n}^{*}=1$. Such a replacement is equivalent to the differentiation of the first boundary integral equation (4) with respect to the co-ordinate $\mathrm{n}$ at the source point $x$. The expression $\tilde{T}_{n}(y)$ denotes shear force for clamped and for simply-supported edges: $\tilde{T}_{n}(y)=T_{n}(y)$ is the shear force (distributed reaction of the support) on the boundary far from the plate corner or $\tilde{T}_{n}(y)=$ $R_{n}(y)$ the distributed reaction along the small fragment of the boundary close to the corner. Because the relation between $\varphi_{s}(y)$ and the deflection is known: $\varphi_{s}(y)=d w(y) / d s$, the angle of rotation $\varphi_{s}(y)$ can be evaluated using a finite difference scheme of the deflection with two or more adjacent nodal values (Guminiak and Sygulski, 2003; Guminiak, 2014).

\subsection{Construction of set of algebraic equations}

The plate boundary is discretized by elements of the constant type. Two approaches of constructing the boundary-domain integral equations are considered. According to the first approach, singular, the collocation points are located exactly on the plate boundary. According to the second approach, non-singular, the collocation points are located outside of the plate boundary (Abaqus, 2005). The plate domain is divided by rectangular subdomains associated with a single collocation point. The set of algebraic equations has the following form:

$\left[\begin{array}{ll}G_{\mathrm{BB}} & -\lambda \cdot G_{\mathrm{B \kappa}} \\ \mathrm{G}_{\kappa \mathrm{B}} & -\lambda \cdot \mathrm{G}_{\kappa \kappa}+\mathrm{I}\end{array}\right] \cdot\left\{\begin{array}{l}\widetilde{\mathrm{B}} \\ \kappa\end{array}\right\}=\left\{\begin{array}{l}0 \\ 0\end{array}\right\}$,

where: the critical force $\mathrm{N}_{\mathrm{cr}}$ is expressed by eigenvalue multiplier $\lambda=N_{x y}=N_{c r}$.

Integration of suitable fundamental functions is done in a local coordinate system ni, si connected with ith boundary element and next, these integrals must be transformed to nk, sk coordinate system, connected with kth element (Guminiak and Sygulski, 2003; Guminiak, 2014). For a non-singular approach, the localization of a collocation point is defined by the parameter $\tilde{\delta}$ which determines the distance from a plate edge or by non-dimensional parameter $\varepsilon=\tilde{\delta} / d$ where $d$ is the element length (Guminiak and Sygulski, 2003; Guminiak, 2014).

The vector of unknowns consist: $\tilde{B}=\left\{B, \varphi_{s}\right\}$ - the vector of boundary independent variables, $\varphi_{s}$ - the vector of additional parameters of the angle of rotation in the tangential direction, which depends on the boundary deflection in case of the free edge and $\kappa$ - the vector specifying curvatures $\kappa_{x y}$ inside a plate domain. The matrix $G_{B B}$ groups boundary integrals dependent on the type of boundary. The matrix $G_{B \kappa}$ includes values of fundamental functions $\mathrm{w}^{*}$ and $\bar{w}^{*}$ established in internal collocation points associated with internal rectangular sub-domains. The matrix $G_{\kappa B}$ groups boundary integrals dependent on the type of boundary (integration from internal collocation points over boundary elements), the matrix $G_{\kappa \kappa}$ groups values of fundamental functions $w^{*}$ and $\bar{w}^{*}$ established in internal collocation points associated with internal rectangular sub-domains (integration from internal collocation points over internal sub-domains) and I is the unit matrix. The second matrix equation (8)2 can be derived by double 
differentiation of the equation (4).

In the present examples only supported plate edges (clamped or simply-supported) are considered, hence the vector $\varphi_{\mathrm{s}}$ has all elements equal to zero thus $\tilde{B}=B$. The analysis of plates with external in-plane loading acting directly along free edges (edges without any constraints) requires a broader analysis. Elimination of boundary variables $B$ and from matrix equation (8) allows to obtain the standard eigenvalue problem (Guminiak and Sygulski, 2003; Guminiak, 2014):

$\{A-\tilde{\lambda} \cdot I\} \cdot \kappa=0$,

where: $\tilde{\lambda}=1 / \lambda$ and

$A=\left\{G_{\kappa \kappa}-\left(G_{\kappa B}\right) \cdot\left[G_{B B}\right]^{-1} \cdot G_{B \kappa}\right\}$.

\subsection{Modes of buckling}

The set of the algebraic equations indispensable to calculate the elements of eigenvector $w$ has the form

$\left[\begin{array}{ll}G_{B B} & 0 \\ G_{w B} & I\end{array}\right] \cdot\left\{\begin{array}{l}B \\ W\end{array}\right\}=\left\{\begin{array}{l}\lambda \cdot G_{B w} \cdot \kappa \\ \lambda \cdot G_{w w} \cdot \kappa\end{array}\right\}$,

where elements of the eigenvector $\kappa$ are obtained after solution of the standard eigenvalue problem (9). The first equation (11)1 is obtained from the first equation of (8) and the second equation (11)2 is got by construction of the boundary integral equations for calculating the plate deflection in internal collocation points. The displacement vector $\mathrm{w}$ can be calculated directly by elimination of boundary variables B (Guminiak and Sygulski, 2003; Guminiak, 2014):

$\mathrm{w}=\lambda \cdot\left[\mathrm{G}_{\mathrm{ww}}-\left(\mathrm{G}_{\mathrm{wB}}\right) \cdot\left[\mathrm{G}_{\mathrm{BB}}\right]^{-1} \cdot \mathrm{G}_{\mathrm{Bw}}\right] \cdot \kappa$.

\section{NUMERICAL EXAMPLES}

The initial stability problem of square plates with various boundary and load conditions is considered. The critical value of the external loading is investigated. The plate properties are: Young modulus $E=205 \mathrm{GPa}$, Poisson ratio $v=0.3$. The following notations are assumed: BEM I - singular formulation of governing boundary-domain integral equations (4) and (5); BEM II - nonsingular formulation of governing boundary-domain integral equations (4) and (5), the collocation point of single boundary element is located outside, near the plate edge, $\varepsilon=\tilde{\delta} / d=0.001$ (Guminiak and Sygulski, 2003); FEM - ABAQUS system with finite elements of the S4R and S8R types are applied (for square plates without holes). The critical force $\mathrm{N}_{\mathrm{cr}}$ is expressed using non-dimensional term: $\widetilde{N}_{c r}=N_{c r} \cdot a^{2} / D$, where $D$ is the plate stiffness and $a$ is dimension of the plate edge.

\subsection{Example 1}

The square plate of dimensions a $=2.0 \mathrm{~m}$, clamped along one edge and simply-supported along other edges (Fig. 3 ) is considered. The plate are subjected to $N_{x y}$ in-plane forces. Plate edges were divided into 128 boundary elements and the number of internal square sub-domains is equal 256 . The number of finite element is equal 1600 . The results of calculation are presented in
Tab. 1 in column a). The first buckling mode is shown in Fig. 4. Column b) contains resuts of calculation for the plate simply supported along all edges.

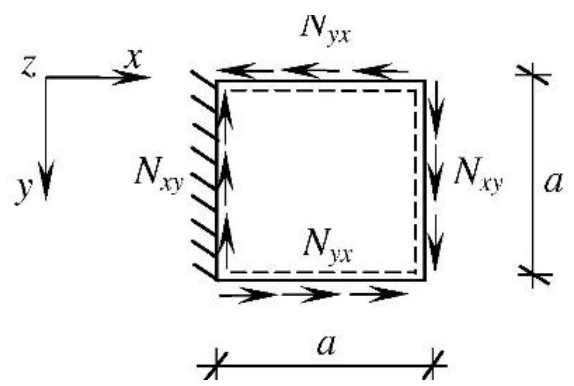

Fig. 3. The plate subjected to in-plane tangential loading

Tab. 1. Critical loading values $\tilde{N}_{\text {cr }}$

\begin{tabular}{|c|c|c|}
\hline$\tilde{N}_{\text {cr }}=\tilde{N}_{x y}$ & a) & b) \\
\hline BEM I & 106.971 & 93.009 \\
\hline BEM II & 106.996 & 93.051 \\
\hline FEM (S4R) & 100.601 & 92.601 \\
\hline FEM (S8R) & 101.323 & 92.799 \\
\hline$[1]$ & - & 92.182 \\
\hline
\end{tabular}

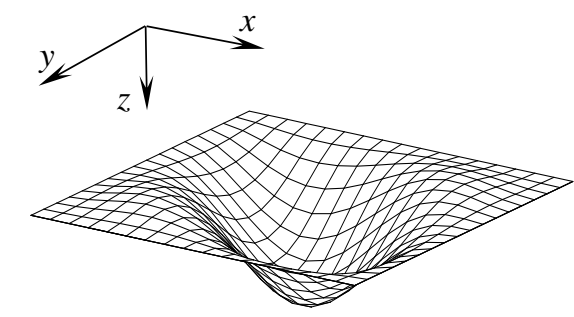

Fig. 4. The first buckling mode for square plate clamped along one edge and simply-supported along other edges

\subsection{Example 2}

The element of the steel welded girder is considered and modelled as the square simply-supported plate. The plate edge dimensions is a $=2.0 \mathrm{~m}$. The plate is subjected to $N_{x}$ and $N_{x y}$ inplane forces with linear and constant distributions respectively (Fig. 5). The finite element discretization is the same as in Example 1. The results of the calculation are presented in Tab. 2. The first buckling mode is shown in Fig. 6 .

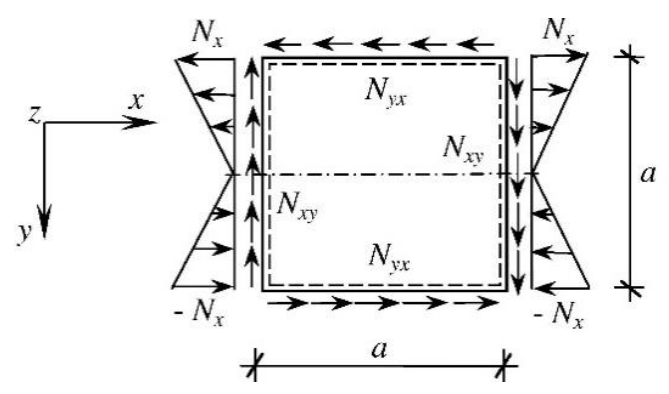

Fig. 5. Considered element of the steel welded girder 
Tab. 2. Critical loading value $\tilde{N}_{\text {cr }}$. Assumed the comparative compressing loading: $\tilde{N}_{x}$

\begin{tabular}{|c|c|c|c|c|c|}
\hline$\tilde{N}_{x y} / \tilde{N}_{x}$ & 0.0 & 0.025 & 0.05 & 0.1 & 0.15 \\
\hline S4R & 251.837 & 250.498 & 247.032 & 236.685 & 224.229 \\
\hline S8R & 250.069 & 248.705 & 245.227 & 235.037 & 222.884 \\
\hline
\end{tabular}
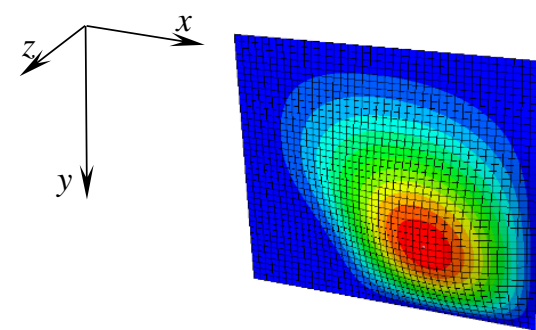

Fig. 6. The first buckling mode for simply-supported square plate $\left(\tilde{N}_{x y} / \tilde{N}_{x}=0.1\right)$

\subsection{Example 3}

The square element of the steel welded girder is considered and modelled as the square plate clamped along one edge and simply-supported along other edges. The plate egde dimension is a $=2.0 \mathrm{~m}$. The plate is subjected to $N_{x}$ and $N_{x y}$ in-plane forces with linear and constant distributions respectively (Fig. 7). The finite element discretization is the same as in Example 1.

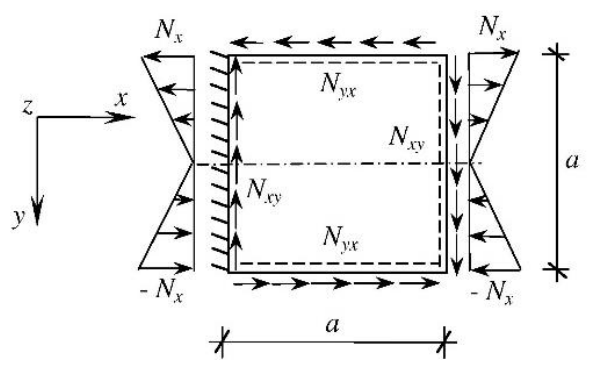

Fig. 7. Considered element of the steel welded girder

The results of the calculation are presented in Tab. 3. The first buckling mode for $\tilde{N}_{x y} / \tilde{N}_{x}=0.1$ is shown in Fig. 8 .

Tab. 3. Critical loading value $\tilde{N}_{\mathrm{cr}}$. Assumed the comparative compressing loading: $\tilde{N}_{x}$

\begin{tabular}{|c|c|c|c|c|c|}
\hline$\tilde{N}_{x y} / \tilde{N}_{x}$ & 0.0 & 0.025 & 0.05 & 0.1 & 0.15 \\
\hline S4R & 259.794 & 256.013 & 251.596 & 241.294 & 229.720 \\
\hline S8R & 261.093 & 257.527 & 253.306 & 243.324 & 231.972 \\
\hline
\end{tabular}

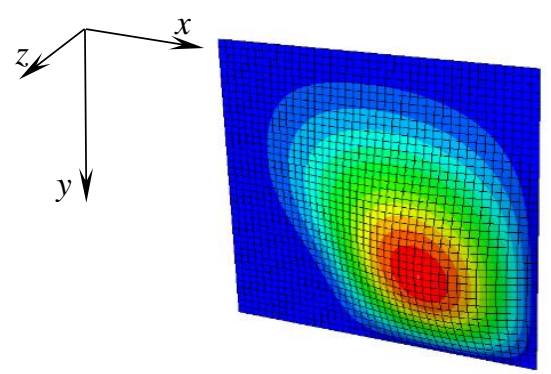

Fig. 8. The first buckling mode for square plate clamped along one edge and simply-supported along other edges $\left(\tilde{N}_{x y} / \tilde{N}_{x}=0.1\right)$

\subsection{Example 4}

The square element of a steel welded girder with the symmetrically square hole is considered and modelled as the square plate. The plate edge dimension is $a=2.0 \mathrm{~m}$. The plate is subjected to $N_{x}$ and $N_{x y}$ in-plane forces with linear and constant distributions respectively (Fig. 9).

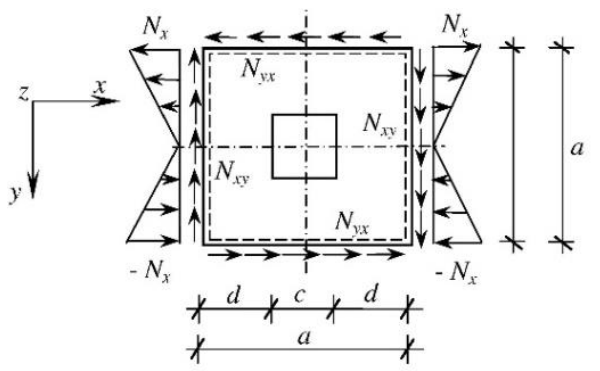

Fig. 9. Considered element of the steel welded girder with square hole

Dimensions of the hole are: $c=0.25 \cdot a, d=0.375 \cdot a$. The total number of finite elements is equal 1745 (Fig. 10).

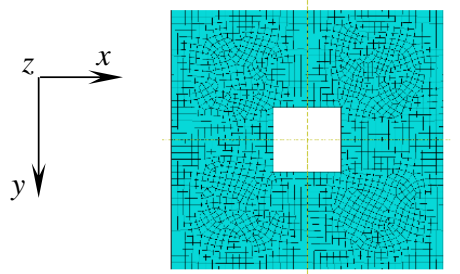

Fig. 10. Considered element of steel welded girder with square hole

The results of the calculation are presented in Tab. 4. The first buckling mode for $\tilde{N}_{x y} / \tilde{N}_{x}=0$ is shown in Fig. 11 .

Tab. 4. Critical loading value $\tilde{N}_{\text {cr. }}$. Assumed the comparative compressing loading: $\tilde{N}_{x}$

\begin{tabular}{|c|c|c|c|c|c|}
\hline$\tilde{N}_{x y} / \tilde{N}_{x}$ & 0.0 & 0.025 & 0.05 & 0.1 & 0.15 \\
\hline FEM1 & 116.411 & 114.277 & 110.584 & 101.183 & 91.164 \\
\hline FEM2 & 115.047 & 112.654 & 108.589 & 98.601 & 88.266 \\
\hline
\end{tabular}

The following designations are assumed: FEM1 - four node rectangular finite elements (S4R) and three node triangle finite elements, with three degrees of freedom per node; FEM2 - eight node rectangular finite elements (S8R) and six node triangle finite elements, with three degree of freedom per node.

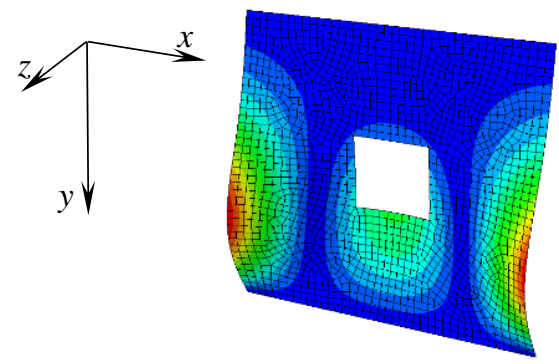

Fig. 11. The first buckling mode for simply-supported square plate with square hole $\left(\tilde{N}_{x y} / \tilde{N}_{x}=0\right)$ 
The first buckling mode for $\tilde{N}_{x y} / \tilde{N}_{x}=0.025$ is shown in Fig. 12 .
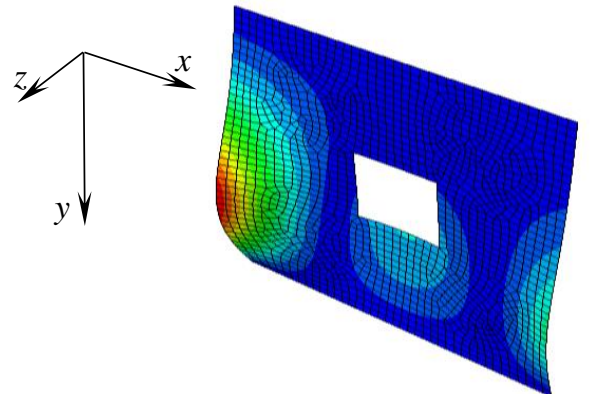

Fig. 12. The first buckling mode for simply-supported square plate with square hole $\left(\tilde{N}_{x y} / \tilde{N}_{x}=0.025\right)$

\subsection{Example 5}

The square element of a steel welded girder with the symmetrically hexagonal hole is considered and modeled as the simplysupported plate. The plate edge dimension is a $=2.0 \mathrm{~m}$. The plate is subjected to tensile $\mathrm{N}_{\mathrm{x}}$ in-plane forces with constant distributions respectively (Fig. 13).

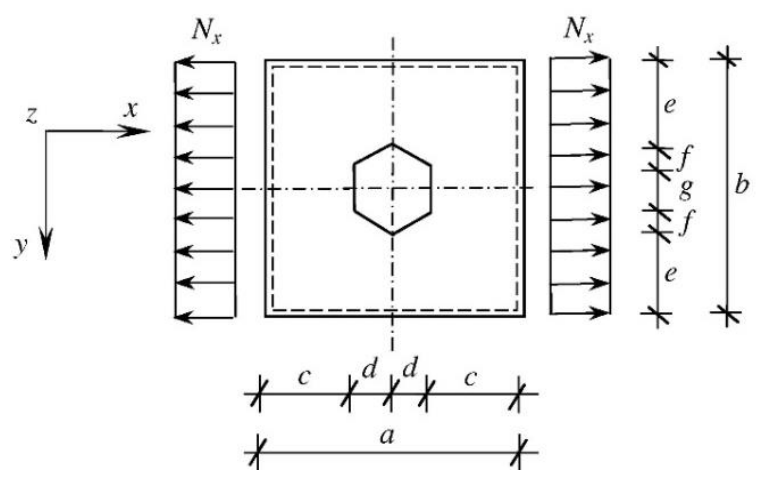

Fig. 13. Considered element of steel welded girder with hexagonal hole

Dimensions of the hole are: $c=0.3268 \cdot a, d=0.1732 \cdot a$, $e=0.3 \cdot a, f=0.1 \cdot a, g=0.2 \cdot a$. The total number of finite elements equals 1685 (Fig. 14).
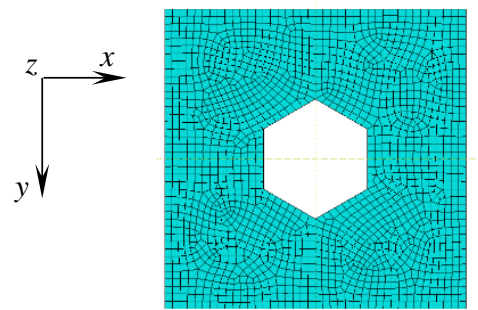

Fig. 14. Considered element of steel welded girder with hexagonal hole

The results of the calculation are presented in Tab. 5 .

Tab. 5. Critical loading value $\tilde{N}_{\mathrm{cr}}=\tilde{N}_{x}$

\begin{tabular}{|c|c|}
\hline FEM1 & FEM2 \\
\hline 488.371 & 479.810 \\
\hline
\end{tabular}

The first buckling mode is shown in Fig. 15.
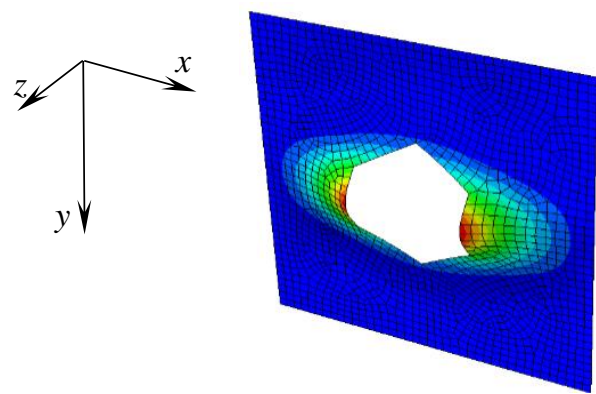

Fig. 15. The first buckling mode for simply-supported square plate with hexagonal hole

\subsection{Example 6}

The 2-meter-long part of the web of the two-span beam was analysed (Fig. 16). It was assumed that the beam was under continuous restraint and was not susceptible to lateral-torsional buckling. There was no shear lag effect. However, the web was with cross section of class 4 (Fig. 17), in which local buckling may occur.

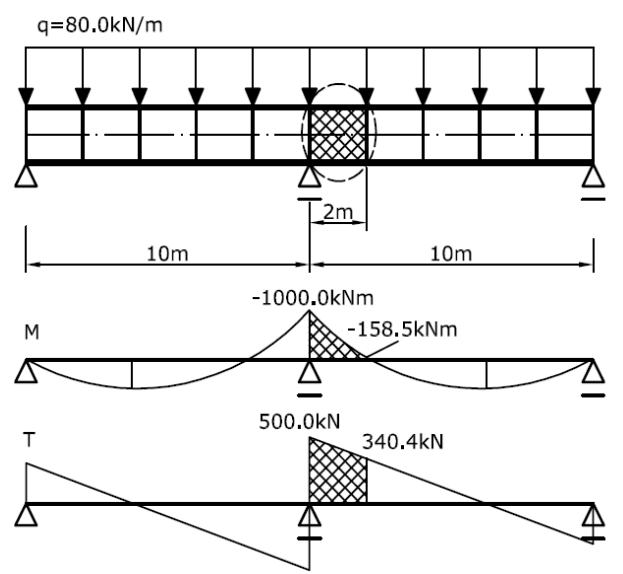

Fig. 16. Analysed steel welded-girder
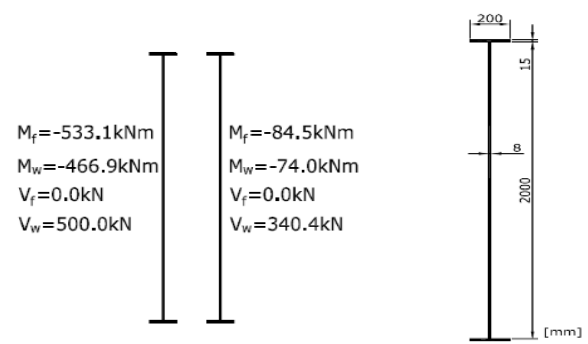

Fig. 17. Analysed steel welded-girder cross-section

For this reason, transverse stiffeners were used every two meters. In this example, the steel square plate was checked for local buckling using the Abaqus finite element system.

The analysed part of the steel welded girder is loaded by bending and shear forces according to Fig. 18. The proportion between shear and bending loading are assumed similarly. 

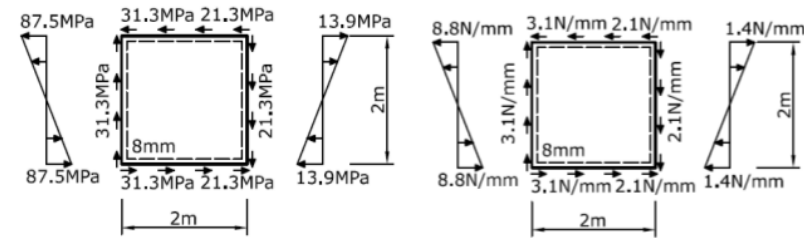

Fig. 18. Analysed steel welded-girder cross-section

This part was divided into 1600 finite elements of S8R type. The boundary conditions are as indicated in Fig. 19. a)

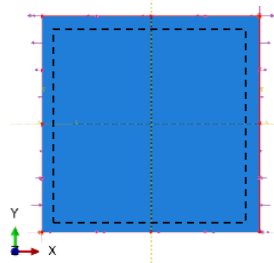

b)

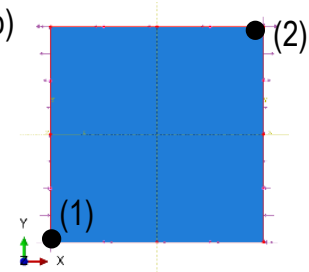

Fig. 19. Boundary conditions: a) blocked transverse displacement $u 3=0$ and $\mathrm{b}$ ) blocked in plane displacements $\mathrm{u} 1=0$ and $\mathrm{u} 2=0$ at selected points (1) and (2)

The results of the calculation are presented in Tab. 6 .

Tab. 6. Critical loading value $N_{\mathrm{cr}}[\mathrm{kN} / \mathrm{m}]$

\begin{tabular}{|c|c|c|}
\hline Only shear & Only bending & Shear and bending \\
\hline 119.53 & 87.533 & 77.407 \\
\hline
\end{tabular}

Due to transverse stiffeners, local buckling did not occur. One can find that there was influence of the shear forces on the eigenvalue.

\section{FINAL CONCLUSIONS}

The analysis of initial stability of steel welded girders was carried out in this paper. The BEM was proposed to solve the simple benchmark test of the plate subjected to shear in-plane forces with constant distribution. The FEM was applied to the complex analysis. The impact of additional tangential in-plane loading cannot be avoided which is confirmed by numerical experiment. Special attention should also be paid to girders with holes. In this case tensile in-plane forces (Example 5) cause compressing in the area near the hole. Note that the problem of plate stability is sensitive on the type of finite element and modeling of boundary conditions. The stress singularities in hole vertices were not specifically considered. The boundary conditions of considered plates are conventional to some extent. It depends on the thickness of the stiffeners, as well as lower and upper flange plates. Example 6 demonstrates the significant effect of shear on the value of critical loading $\mathrm{N}_{\mathrm{cr}}$ which is shown in Tab. 6 (the critical load was reduced by more than 10 percent). Therefore good estimation of critical force is primary importance for the reasonable designing of the plane girder.

\section{REFERENCES}

1. Abaqus, Abaqus Manuals. (2005), Inc. Providence.

2. Biegus A., Kowal A. (2013), Collapse of halls made from coldformed steel sheets, Engineering Failure Analysis, 31, 189-194.

3. Chinnaboon B., Chucheepsakul S., Katsikadelis J.T. (2007), A BEM-bases Meshless Method for Buckling Analysis of Elastic Plates with Various Boundary Conditions, Journal of Structural Stability and Dynamics, 1(7), 81-89.

4. Chybiński M. (2015), Load capacity and stability of steel thin-walled beams with local stiffening elements, The Poznan University of Technology Publishing House, Poznan (in Polish).

5. Chybinski M., Rzeszut K., Garstecki A. (2013), Snap-through phenomenon of imperfect steel structures, Proceedings of International Conference Design, Fabrication and Economy of Metal Structures, Miskolc, Hungary, Springer-Verlag.

6. Garstecki A., Rzeszut K. (2009), Modeling of initial geometrical imperfections in stability analysis of thin-walled structures, Journal of Theoretical and Applied Mechanics, 47(3), 667-684.

7. Gosowski B. (1999), Spatial bucling of thin-walled steel-construction beam-columns with discrete bracings, Journal of Constructional Steel Research, 52(3), 293-317.

8. Guminiak M. (2014), An Alternative Approach of Initial Stability Analysis of Kirchhoff Plates by the Boundary Element Method, Engineering Transactions, 62(1), 33-59.

9. Guminiak M., Sygulski R. (2003), Initial stability of thin plates by the Boundary Element Method, Stability of Structures X Symposium, Zakopane, Poland, eds.: K. Kowal-Michalska, Z. Kołakowski.

10. Litewka B., Sygulski R. (2010), Application of the fundamental solutions by Ganowicz in a static analysis of Reissner's plates by the boundary element method, Engineering Analysis with Boundary Elements, 34(12), 1072-1081.

11. Marcinowski J. (2007), Stability of relatively deep segments of spherical shells loaded by external pressure, Thin-Walled Structures, 45(10-11), 906-910.

12. Rakowski G., Kacprzyk Z. (2005), The Finite Element Method in Stuctural Mechanics, The Warsaw University of Technology Publishing House, Warsaw (in Polish).

13. Rzeszut K., Garstecki A. (2011), Thin-walled structures with slotted connections in stability problems, Thin-Walled Structures, 49, 674-681.

14. Rzeszut K., Garstecki A. (2013), Stability of thin-walled structures accounting for initial imperfections and clearances, in.: Statics, dynamics and stability of structures, Vol. 3, 127-146.

15. Rzeszut K., Polus L. (2013), Classes of Cross-Sections of Steel Structrural Elements in the Fire Situation, Procedia Engineering, $57,967-976$.

16. Shi G. (1990), Flexural vibration and buckling analysis of orthotropic plates by the Boundary Element Method, Journal of Thermal Stresses, 26(12), 1351-1370.

17. Timoshenko S., Woynowsky-Krieger S. (1962), Theory of elstic stability, Arkady, Warszawa. 